\title{
A Novel Approach for the Synthesis of 5-Substituted-1H-tetrazoles
}

\author{
Batool Akhlaghinia* and Soodabeh Rezazadeh \\ Department of Chemistry, Faculty of Sciences, Ferdowsi University of Mashhad, \\ 9177948974 Mashhad, Iran
}

\begin{abstract}
Uma série de $1 \mathrm{H}$-tetrazóis-5-substituídos $\left(\mathrm{RCN}_{4} \mathrm{H}\right)$ foi sintetizada pela reação de cicloadição de diferentes aril e alquil nitrilas com azida de sódio em DMSO, usando $\mathrm{CuSO}_{4} \cdot 5 \mathrm{H}_{2} \mathrm{O}$ como catalisador. Uma grande variedade de aril nitrilas sofreu cicloadição [3+2], obtendo-se os correspondentes tetrazóis sob condições reacionais brandas. O catalisador utilizado é obtido facilmente e ambientalmente correto. Tempos de reação curtos, bons a excelentes rendimentos, processo seguro e simples tornam este método uma contribuição atrativa e útil à presente síntese orgânica de $1 H$-tetrazóis-5-substituídos.
\end{abstract}

A series of 5-substituted-1H-tetrazoles $\left(\mathrm{RCN}_{4} \mathrm{H}\right)$ have been synthesized by cycloaddition reaction of different aryl and alkyl nitriles with sodium azide in DMSO using $\mathrm{CuSO}_{4} \cdot 5 \mathrm{H}_{2} \mathrm{O}$ as catalyst. A wide variety of aryl nitriles underwent [3+2] cycloaddition to afford tetrazoles under mild reaction conditions in good to excellent yields. The catalyst used is readily available and environmentally friendly. Short reaction times, good to excellent yields, safe process and simple workup make this method an attractive and useful contribution to present organic synthesis of 5-substituted- $1 H$-tetrazoles.

Keywords: 5-substituted- $1 \mathrm{H}$-tetrazole, arylnitriles, sodium azide, $\mathrm{CuSO}_{4} \cdot 5 \mathrm{H}_{2} \mathrm{O}$, cycloaddition reaction

\section{Introduction}

The chemistry of heterocyclic compounds has been an interesting field of study for a long time. Tetrazoles as a group of heterocyclic compounds are reported to possess a broad spectrum of biological activities such as antibacterial, ${ }^{1}$ antifungal, ${ }^{2}$ antiviral, ${ }^{3-5}$ analgesic, ${ }^{6,7}$ anti-inflammatory, ${ }^{8-10}$ antiulcer ${ }^{11}$ and antihypertensive ${ }^{12}$ activities. Also, 5 -substituted- $1 H$-tetrazoles can function as lipophilic spacers and carboxylic acid surrogates, ${ }^{13}$ specialty explosives ${ }^{14,15}$ and information recording systems in materials ${ }^{16}$ ligands, and precursors of a variety of nitrogen containing heterocycles in coordination chemistry. ${ }^{17,18}$ Since $1901,{ }^{19}$ conventional synthesis of 5 -substituted$1 H$-tetrazoles has been reported to proceed via $[3+2]$ cycloaddition of azide with nitriles. This procedure suffers from numerous drawbacks including use of expensive and toxic metal organic azide ${ }^{20}$ highly moisture-sensitive reaction conditions, strong Lewis acid, ${ }^{21}$ and hydrazoic acid. ${ }^{22}$ The "click" chemistry approach utilizing zinc

*e-mail: akhlaghinia@um.ac.ir catalysis in aqueous solution is a magnificent improvement over latter methods, ${ }^{23,24}$ but occasionally still requires the tedious and time-consuming removal of zinc salts from the acidic products. Stoichiometric amounts of inorganic salts and metal complexes ${ }^{25}$ as catalysts, use of $\mathrm{TMSN}_{3}$ and $\mathrm{TBAF}^{26}$ instead of metal salts under solvent-free conditions in micellar media and ionic liquids, and use of various catalysts ${ }^{27-31}$ such as $\mathrm{BF}_{3} \cdot \mathrm{OEt}_{2},{ }^{21} \mathrm{Pd}(\mathrm{OAc})_{2} / \mathrm{ZnBr}_{2},{ }^{27}$ $\mathrm{Yb}(\mathrm{OTf})_{3},{ }^{28} \mathrm{Zn}(\mathrm{OTf})_{3},{ }^{29} \mathrm{AlCl}_{3},{ }^{30}$ and $\mathrm{Pd}\left(\mathrm{PPh}_{3}\right)_{4}{ }^{31}$ were also employed for the same purpose. However, a drawback of these homogeneous catalytic processes lies in the tedious separation and recovery of the catalysts. Recently, several heterogeneous catalytic systems ${ }^{32-40}$ using nanocrystalline $\mathrm{ZnO}, \mathrm{Zn} / \mathrm{Al} \mathrm{HT},{ }^{32} \mathrm{Zn}$ hydroxyapatite, ${ }^{33} \mathrm{Cu}_{2} \mathrm{O},{ }^{34,35} \mathrm{Sb}_{2} \mathrm{O}_{3}$, $\mathrm{FeCl}_{3} / \mathrm{SiO}_{2},{ }^{36} \mathrm{CdCl}_{2},{ }^{37} \mathrm{BaWO}_{4}, \gamma-\mathrm{Fe}_{2} \mathrm{O}_{3},{ }^{38} \mathrm{ZnS},{ }^{39}$ and natural natrolite zeolite ${ }^{40}$ were reported. Metal-modified montmorillonites and zeolite were reported widely, and many metals, such as $\mathrm{Cu}, \mathrm{Zn}, \mathrm{Mn}, \mathrm{Fe}, \mathrm{Cu}, \mathrm{V}, \mathrm{Mo}, \mathrm{Al}$ and Co ${ }^{41-48}$ were commonly used to improve the catalytic abilities of montmorillonites. These methods require a large excess of sodium azide, longer reaction time, and expensive metals. Moreover, the cycloaddition is too slow 
to be synthetically useful except when strong electronwithdrawing groups activate the nitrile compounds.

Today's stringent environmental and legislative concerns demand for the green methods that reduce the use of toxic and corrosive reagents and stop the formation of inorganic wastes. ${ }^{49}$ However, it has been also observed that the catalysts employed are not always eco-friendly and because of this, serious environmental pollution often results. Therefore, in the area of green synthesis, the development of environmentally friendly alternatives is desirable for the synthesis of tetrazoles. ${ }^{50}$

Recently, we have studied the application of cupric sulfate pentahydrate in the trimethylsilylation of alcohols and phenols, ${ }^{51}$ and perceived that cupric sulfate pentahydrate as a mild Lewis acid, which is readily available, might be a useful catalyst for the synthesis of 5-substituted-1Htetrazoles. In this paper we report a new process for the synthesis of 5-substituted $1-H$-tetrazoles using cupric sulfate pentahydrate as a safe, environmentally benign, and inexpensive catalyst.

\section{Experimental}

The products were purified by column chromatography. The purity determinations of the products were accomplished by TLC on silica gel polygram STL G/UV 254 plates. The melting points of products were determined with an Electrothermal Type 9100 melting point apparatus. The FT-IR spectra were recorded on an Avatar 370 FT-IR Thermo Nicolet spectrometer. The NMR spectra were provided on a Bruker Avance 100 and $400 \mathrm{MHz}$ instrument. All of the products were known compounds and characterized by the IR, ${ }^{1} \mathrm{H}$ NMR and ${ }^{13} \mathrm{C}$ NMR spectra and comparison of their melting points with known compounds. Elemental analyses were performed using a Elementar, Vario EL III and Thermofinnigan Flash EA 1112 Series instrument. Mass spectra were recorded with Agilent Technologies (HP) 5973 Network Mass Selective Detector and Shimadzu GC-MS-QP5050 instruments at $70 \mathrm{eV}$.

Typical procedure for the preparation of 5-phenyl-1Htetrazole (1)

Sodium azide $(0.0650 \mathrm{~g}, 1 \mathrm{mmol})$ and cupric sulfate pentahydrate $(0.0050 \mathrm{~g}, 2 \mathrm{~mol} \%)$ were added to a solution of benzonitrile $(0.1031 \mathrm{~g}, 1 \mathrm{mmol})$ in DMSO $(2 \mathrm{~mL})$ with stirring at room temperature. The reaction temperature was raised up to $140{ }^{\circ} \mathrm{C}$ for $1 \mathrm{~h}$. The progress of the reaction was monitored by TLC. After completion, the reaction mixture was cooled and treated with $10 \mathrm{~mL} \mathrm{HCl}\left(4 \mathrm{~mol} \mathrm{~L}^{-1}\right)$ and then with $10 \mathrm{~mL}$ EtOAc. The resultant organic layer was separated, washed with $2 \times 10 \mathrm{~mL}$ distilled water, dried over anhydrous sodium sulfate, and concentrated to give the crude solid 5-phenyl-1H-tetrazole (1). The crude product was recrystallized from $n$-hexane:ethylacetate (1:1) obtaining $0.1430 \mathrm{~g}$ of colourless crystals ( $98 \%$ yield). The other products( 2-17) were also, recrystallized from $n$-hexane:ethyl acetate $(1: 1)$.

Characterization data for representative compounds

\section{5-Phenyl-1H-tetrazole (1)}

mp 214-216 ${ }^{\circ} \mathrm{C}$ (Lit. 214-216 ${ }^{\circ} \mathrm{C}$ ); ${ }^{52}$ FT-IR (KBr) $v_{\max } / \mathrm{cm}^{-1}: 3125,3043,2982,2913,2835,2765,2692,2606$, 2557, 2488, 1613, 1563, 1485, 1465, 1409, 1163, 1056, 726, 703, 687; ${ }^{1} \mathrm{H}$ NMR (400 MHz, DMSO- $\left.d_{6}\right) \delta 7.61(\mathrm{~s}$, $3 \mathrm{H}, \mathrm{Ph}), 8.05$ (s, 2H, Ph); ${ }^{13} \mathrm{C}$ NMR $\left(100 \mathrm{MHz}, \mathrm{DMSO}-d_{6}\right)$ $\delta$ 124.6, 127.4, 129.9, 131.7, 155.7; CHN $\left(\mathrm{C}_{7} \mathrm{H}_{6} \mathrm{~N}_{4}\right)$ calc. (\%): C (57.5), H (4.1), N (38.3); found (\%): C (56.4), $\mathrm{H}$ (4.1), N (37.6).

5-(4-Bromophenyl)-1H-tetrazole (2) mp 264-265 ${ }^{\circ} \mathrm{C}$ (Lit. $265{ }^{\circ} \mathrm{C}$ ) $;^{53}$ FT-IR (KBr) $\mathrm{v}_{\max } / \mathrm{cm}^{-1}: 3089,3063,2996,2900,2844,2761,2729,2633$, 1652, 1604, 1560, 1482, 1431, 1405, 1157, 1076, 1054, 1018, 829, 744, 502; ${ }^{1} \mathrm{H}$ NMR (100 MHz, DMSO- $\left.d_{6}\right) \delta$ 7.80 (d, 2H, J 9.5 Hz, Ph), 7.95 (d, 2H, J 9.5 Hz, Ph); $\mathrm{CHN}\left(\mathrm{C}_{7} \mathrm{H}_{5} \mathrm{BrN}_{4}\right)$ calc. (\%): $\mathrm{C}$ (37.3), H (2.2), N (24.9); found (\%): $\mathrm{C}$ (37.2), $\mathrm{H}(2.1), \mathrm{N}(24.6) ; \mathrm{MS}(\mathrm{EI}): \mathrm{m} / \mathrm{z}(\%)$ $226\left[\mathrm{M}^{+}+2\right], 224\left[\mathrm{M}^{+}\right], 198\left[\left(\mathrm{M}^{+}+2\right)-\mathrm{N}_{2}\right], 196(100)\left[\mathrm{M}^{+}-\right.$ $\left.\mathrm{N}_{2}\right], 185\left[\left(\mathrm{M}^{+}+2\right)-\mathrm{N}_{3}\right], 183\left[\mathrm{M}^{+}-\mathrm{N}_{3}\right]$.

\section{5-(4-Chlorophenyl)-1H-tetrazole (3)}

mp 261-262 ${ }^{\circ} \mathrm{C}$ (Lit. 261-263 ${ }^{\circ} \mathrm{C}$ ); ${ }^{48}$ FT-IR (KBr) $v_{\max } / \mathrm{cm}^{-1}: 3092,3060,3007,2978,2907,2851,2725,2622$, 2537, 2471, 1609, 1564, 1486, 1435, 1160, 1096, 1053, 1020, 990, 833, 745, 508; ${ }^{1} \mathrm{H}$ NMR (400 MHz, DMSO- $\left.d_{6}\right)$ $\delta 7.68(\mathrm{~d}, 2 \mathrm{H}, J 8.4 \mathrm{~Hz}, \mathrm{Ph}), 8.05$ (d, 2H, J 8.8 Hz, Ph). ${ }^{13} \mathrm{C}$ NMR (100 MHz, DMSO- $\left.d_{6}\right) \delta 123.5,129.2,130.0$, 136.4, 155.3; $\mathrm{CHN}\left(\mathrm{C}_{7} \mathrm{H}_{5} \mathrm{ClN}_{4}\right)$ calc. (\%): $\mathrm{C}(46.6), \mathrm{H}(2.7)$, $\mathrm{N}$ (31.0); found (\%): C (46.5), H (2.7), N (31.0).

4-(1H-Tetrazol-5-yl)benzonitrile (4)

mp 190-191 ${ }^{\circ} \mathrm{C}$ (Lit. $\left.192{ }^{\circ} \mathrm{C}\right) ;{ }^{54} \mathrm{FT}-\mathrm{IR}(\mathrm{KBr}) v_{\max } / \mathrm{cm}^{-1}$ : 3150, 3092, 3013, 2928, 2861, 2758, 2610, 2231, 1585, 1560, 1494, 1433, 1279, 1153, 1014, 976, 944, 850, 749, 554; ${ }^{1} \mathrm{H}$ NMR (100 MHz, CD 3 CN) $\delta 7.90$ (d, 2H, J 7.5 Hz, $\mathrm{Ph}), 8.20$ (d, 2H, J 7.5 Hz, Ph).

\section{5-(4-Nitrophenyl)-1H-tetrazole (5)}

mp 218-219 ${ }^{\circ} \mathrm{C}$ (Lit. 219-220 ${ }^{\circ} \mathrm{C}$ ) $;^{55}$ FT-IR (KBr) $v_{\max } / \mathrm{cm}^{-1}: 3448,3334,3235,3109,3080,2974,2900,2819$, 
2659, 1562, 1532, 1488, 1357, 1340, 1315, 1143, 1106, 995, 867, 853, 730, 710; ${ }^{1} \mathrm{H}$ NMR (400 MHz, DMSO- $d_{6}$ ) $\delta 8.31(\mathrm{~d}, 2 \mathrm{H}, J 8.4 \mathrm{~Hz}, \mathrm{Ph}), 8.46(\mathrm{~d}, 2 \mathrm{H}, J 8.8 \mathrm{~Hz}, \mathrm{Ph})$; ${ }^{13} \mathrm{C}$ NMR (100 MHz, DMSO- $\left.d_{6}\right) \delta 125.1,128.6,131.0$, 149.2, 155.9.

\section{4-Nitro-2-(1 H-tetrazol-5-yl)benzenamine (6)} mp 268-270 ${ }^{\circ} \mathrm{C}$ (Lit. $270-271{ }^{\circ} \mathrm{C}$ ) $;{ }^{56}$ FT-IR (KBr) $v_{\max } / \mathrm{cm}^{-1}: 3411,3321,3199,3084,2937,1645,1616,1572$, 1477, 1325, 1278, 1141, 1041, 910, 831, 751, 722; ${ }^{1} \mathrm{H}$ NMR $\left(400 \mathrm{MHz}, \mathrm{DMSO}-d_{6}\right) \delta 7.00(\mathrm{~d}, 1 \mathrm{H}, J 9.2 \mathrm{~Hz}, \mathrm{Ph}), 7.94$ (br s, NH), 8.10 (dd, 1H, J 9.2, J $2.4 \mathrm{~Hz}, \mathrm{Ph}$ ), 8.81 (d, 1H, $J 2.4 \mathrm{~Hz}, \mathrm{Ph}) .{ }^{13} \mathrm{C}$ NMR (100 MHz, DMSO- $\left.d_{6}\right) \delta 104.4$, 116.3, 126.1, 127.6, 136.3, 153.0, 154.4; $\mathrm{CHN}\left(\mathrm{C}_{7} \mathrm{H}_{6} \mathrm{~N}_{6} \mathrm{O}_{2}\right)$ calc. (\%): C (40.7), H (2.9), N (40.7); found (\%): C (39.7), $\mathrm{H}(3.3), \mathrm{N}(38.1)$.

\section{5-(4-Ethoxyphenyl)-1 $H$-tetrazole (7)}

mp 234-235 ${ }^{\circ} \mathrm{C}$; FT-IR (KBr) $v_{\max } / \mathrm{cm}^{-1}: 3145,3101$, 3060, 2986, 2921, 2868, 2737, 2647, 1613, 1505, 1470, 1394, 1293, 1262, 1189, 1056, 1041, 923, 827, 751, 653, 522 ; ${ }^{1} \mathrm{H}$ NMR (100 MHz, acetone- $\left.d_{6}\right) \delta 1.40(\mathrm{t}, 3 \mathrm{H}, J 5 \mathrm{~Hz}$, -OEt), 4.20 (q, 2H, -OEt), 7.15 (d, 2H, J 9.5 Hz, Ph), 8.07 (d, $2 \mathrm{H}, J 9.5 \mathrm{~Hz}, \mathrm{Ph}) ; \mathrm{CHN}\left(\mathrm{C}_{9} \mathrm{H}_{10} \mathrm{~N}_{4} \mathrm{O}\right.$ ) calc. (\%): $\mathrm{C}(56.8)$, $\mathrm{H}$ (5.2), N (29.5); found (\%): C (57.0), H (4.9), N (30.3).

\section{5-(3,5-Dimethoxyphenyl)- $1 \mathrm{H}$-tetrazole (8)}

mp 204-205 ${ }^{\circ} \mathrm{C}$ (Lit. 204-206 ${ }^{\circ} \mathrm{C}$ ); ${ }^{57}$ FT-IR (KBr) $v_{\text {max }} / \mathrm{cm}^{-1}: 3129,3064,3011,2975,2941,2843,2757,2712$, 2634, 1605, 1562, 1480, 1430, 1287, 1208, 1162, 1167, $1054,827,747 ;{ }^{1} \mathrm{H}$ NMR (400 MHz, DMSO- $\left.d_{6}\right) \delta 3.84$ (s, $6 \mathrm{H},-\mathrm{OMe}), 6.73$ (t, $1 \mathrm{H}, J 2 \mathrm{~Hz}, \mathrm{Ph}), 7.21$ (d, 2H, J $2 \mathrm{~Hz}$, $\mathrm{Ph}), 16.91(\mathrm{br} \mathrm{s}, \mathrm{NH}) ;{ }^{13} \mathrm{C}$ NMR (100 MHz, DMSO- $\left.d_{6}\right) \delta$ 56.0, 103.4, 105.3, 125.7, 156.3, 161.5; MS (EI): $\mathrm{m} / z(\%)$ $207[\mathrm{M}+\mathrm{H}], 149(100)\left[\mathrm{M}^{+}-2 \mathrm{~N}_{2}\right]$.

\section{5-m-Tolyl-1 $\mathrm{H}$-tetrazole (9)}

mp 149.5-150 ${ }^{\circ} \mathrm{C}$ (Lit. $151-152{ }^{\circ} \mathrm{C}$ ) ${ }^{27}$ FT-IR (KBr) $v_{\max } / \mathrm{cm}^{-1}: 3120,3061,2979,2917,2871,2746,2611,2490$, $1728,1605,1565,1486,1463,1150,1060,1038,802,741$, 705,$687 ;{ }^{1} \mathrm{H}$ NMR $\left(100 \mathrm{MHz}, \mathrm{CD}_{3} \mathrm{CN}\right) \delta 2.43\left(\mathrm{~s}, 3 \mathrm{H}, \mathrm{CH}_{3}\right)$, 7.40-7.90 (m, 4H, Ph); $\mathrm{CHN}\left(\mathrm{C}_{8} \mathrm{H}_{8} \mathrm{~N}_{4}\right)$ : calc. (\%) C (59.9), $\mathrm{H}(5.0), \mathrm{N}$ (34.9); found (\%): C (60.0), H (5.0), N (34.7).

\section{4-(1H-Tetrazol-5-yl)phenol (10)}

mp 233-234 ${ }^{\circ} \mathrm{C}$ ( Lit. 234-235 ${ }^{\circ} \mathrm{C}$ ) ${ }^{55}$ FT-IR (KBr) $v_{\max } / \mathrm{cm}^{-1}: 3252,3101,3066,3019,3000-2200,1615$, 1599, 1511, 1466, 1413, 1282, 832, 752, 514; ' ${ }^{1} \mathrm{H}$ NMR $\left(400 \mathrm{MHz}, \mathrm{DMSO}-d_{6}\right) \delta 6.97(\mathrm{~d}, 2 \mathrm{H}, J 8.4 \mathrm{~Hz}, \mathrm{Ph}), 7.87$ (d, $2 \mathrm{H}, J 8.8 \mathrm{~Hz}, \mathrm{Ph}$ ), 10.20 (br s, OH); ${ }^{13} \mathrm{C}$ NMR $(100 \mathrm{MHz}$, DMSO- $\left.d_{6}\right) \delta 115.0,116.6,129.2,155.2,160.5$.
5-(Phenanthren-9-yl)-1H-tetrazole (11) mp 241-242 ${ }^{\circ} \mathrm{C}$ (Lit. $243-244{ }^{\circ} \mathrm{C}$ ) $;^{58}$ FT-IR (KBr) $v_{\max } / \mathrm{cm}^{-1}: 3105,3076,3016,2978,2878,2830,2724,2686$, 2622, 2590, 2520, 2478, 1612, 1565, 1450, 1399, 1246, 1112, 1053, 1038, 992, 934, 771, 737, 721, 424; ' ${ }^{\mathrm{H}} \mathrm{NMR}$ $\left(100 \mathrm{MHz}, \mathrm{DMSO}-d_{6}\right) \delta 7.79-7.82(\mathrm{~m}, 4 \mathrm{H}, \mathrm{Ph}), 8.08-8.20$ (m, 1H, Ph), 8.40-8.52 (m, 2H, Ph), 8.92-9.10 (m, 2H, Ph); CHN $\left(\mathrm{C}_{15} \mathrm{H}_{10} \mathrm{~N}_{4}\right)$ calc. (\%): C (73.1), H (4.0), N (22.7); found (\%): C (73.1), H (3.9), N (22.3); MS (EI): $\mathrm{m} / z$ (\%) $246\left[\mathrm{M}^{+}\right], 218(100)\left[\mathrm{M}^{+}-\mathrm{N}_{2}\right], 190\left[\mathrm{M}^{+}+2 \mathrm{~N}_{2}\right]$.

\section{5-(Thiophen-2-yl)-1H-tetrazole (12)}

mp 205-207 ${ }^{\circ} \mathrm{C}$ (Lit. 205-207 ${ }^{\circ} \mathrm{C}$ ) $;{ }^{59}$ FT-IR (KBr) $v_{\max } / \mathrm{cm}^{-1}: 3109,3074,2974,2891,2780,2722,2628,2569$, 2500, 2456, 1830, 1595, 1503, 1411, 1233, 1139, 1046, $962,853,740,719 ;{ }^{1} \mathrm{H}$ NMR (100 MHz, $\left.\mathrm{CD}_{3} \mathrm{CN}\right) \delta 7.20-$ 7.30 (m, $1 \mathrm{H}$, tiophen), 7.67-7.80 (m, 2H, tiophen); $\mathrm{CHN}$ $\left(\mathrm{C}_{5} \mathrm{H}_{4} \mathrm{~N}_{4} \mathrm{~S}\right.$ ) calc.(\%): C (39.4), H (2.6), N (36.8), S (21.1); found (\%): C (39.1), H (2.6), N (37.0), S (21.4); MS (EI) $m / z(\%) 152\left[\mathrm{M}^{+}\right], 124(100)\left[\mathrm{M}^{+}-\mathrm{N}_{2}\right], 97\left[\mathrm{M}^{+}-2 \mathrm{~N}_{2}\right]$.

4-(1H-Tetrazol-5-yl)pyridine (13)

mp 255-258 ${ }^{\circ} \mathrm{C}$ (Lit. $255-258{ }^{\circ} \mathrm{C}$ ) $;{ }^{52}$ FT-IR (KBr) $v_{\text {max }} / \mathrm{cm}^{-1}: 3485,3264,3099,3035,2966,1624,1529,1435$, 1388, 1123, 1096, 1042, 1022, 845, 730, 674, 593, 465.

\section{2-(1H-Tetrazol-5-yl)pyridine (14)}

mp 211-213 ${ }^{\circ} \mathrm{C}$ (Lit. $210-213{ }^{\circ} \mathrm{C}$ ) $;{ }^{48}$ FT-IR (KBr) $v_{\text {max }} / \mathrm{cm}^{-1}: 3088,3060,2959,2929,2864,2737,2692,2622$, $2582,1728,1602,1557,1483,1449,1405,1284,1158,1068$, 1024, 955, 795, 743, 726, 703, 637, 496; ' $\mathrm{H}$ NMR (400 MHz, DMSO- $d_{6}$ ) $\delta 7.65$ (s, $\left.1 \mathrm{H}, \mathrm{Py}\right), 8.10$ (s, $\left.1 \mathrm{H}, \mathrm{Py}\right), 8.24$ (d, $1 \mathrm{H}, J 6.4 \mathrm{~Hz}, \mathrm{Py}), 8.81$ (s, 1H, Py); ${ }^{13} \mathrm{C}$ NMR $(100 \mathrm{MHz}$, DMSO- $\left.d_{6}\right) \delta 123.1,126.7,138.7,144.0,150.6,155.3$.

\section{5-Isobutyl-1 $H$-tetrazole (15)}

mp 52-54 ${ }^{\circ} \mathrm{C}$ (Lit. 53.5-54 $\left.{ }^{\circ} \mathrm{C}\right) ;{ }^{60} \mathrm{FT}-\mathrm{IR}(\mathrm{KBr}) \mathrm{v}_{\max } / \mathrm{cm}^{-1}$ : 3089, 3063, 2971, 2901, 2845, 2765, 2729, 2633, 1605, $1482,1454,1430,1156,1075,1053,1017,990,829,772$, 743, 502.

\section{5-Isopentyl-1H-tetrazole (16)}

mp $94^{\circ} \mathrm{C}$ (Lit. $\left.95-96^{\circ} \mathrm{C}\right),{ }^{60} \mathrm{FT}-\mathrm{IR}(\mathrm{KBr}) v_{\max } / \mathrm{cm}^{-1}: 2962$, 2931, 2874, 2709, 2618, 2482, 1867, 1583, 1553, 1469 , $1404,1110,1048,772 ;{ }^{1} \mathrm{H}$ NMR $\left(100 \mathrm{MHz}, \mathrm{CDCl}_{3}\right) \delta 1.00$ (d, $\left.6 \mathrm{H}, J 5 \mathrm{~Hz}, 2 \mathrm{CH}_{3}\right), 1.40-2.05\left(\mathrm{~m}, 3 \mathrm{H},-\mathrm{CH}-,-\mathrm{CH}_{2}-\right.$ ), $3.10\left(\mathrm{t}, 2 \mathrm{H}, J 6 \mathrm{~Hz},-\mathrm{CH}_{2}-\right.$ ).

\section{5-Benzyl-1 $\mathrm{H}$-tetrazole (17)}

mp 117-119 ${ }^{\circ} \mathrm{C}$ (Lit. $118-120{ }^{\circ} \mathrm{C}$ ); ${ }^{61}$ FT-IR (KBr) $v_{\text {max }} / \mathrm{cm}^{-1}: 3109,3031,2984,2945,2863,2778,2704$, 
2594, 1768, 1707, 1638, 1549, 1533, 1494, 1457, 1241, $1108,1074,772,734,695 ;{ }^{1} \mathrm{H}$ NMR (100 MHz, $\left.\mathrm{CD}_{3} \mathrm{CN}\right)$ $\delta 4.30\left(\mathrm{~s}, 2 \mathrm{H},-\mathrm{CH}_{2}-\right), 7.31(\mathrm{~s}, 5 \mathrm{H}, \mathrm{Ph}) ; \mathrm{CHN}\left(\mathrm{C}_{8} \mathrm{H}_{8} \mathrm{~N}_{4}\right)$ calc. (\%): C (60.0), H (5.0), N (34.9); found (\%): C (60.6), $\mathrm{H}$ (4.9), N (34.6).

\section{Results and Discussion}

In a typical experiment, reaction of benzonitrile with sodium azide in the presence of $\mathrm{CuSO}_{4} \cdot 5 \mathrm{H}_{2} \mathrm{O}$ was first studied under various reaction parameters. The general reaction is outlined in Scheme 1 and the results are summarized in Table 1.

Generally, with the catalysis of $\mathrm{CuSO}_{4} \cdot 5 \mathrm{H}_{2} \mathrm{O}$ in DMF (Table 1, entries 2-4), the reaction gave good to excellent yields, whereas in the absence of catalyst 5-phenyl-1Htetrazole (1) was obtained in $40 \%$ yield (Table 1, entry 1). On the basis of data in Table 1, the best amount of $\mathrm{CuSO}_{4} \cdot 5 \mathrm{H}_{2} \mathrm{O}$ as catalyst is $2 \mathrm{~mol} \%$ (Table 1, entries 2-4 and entry 12). In an effort to develop better reaction conditions, different solvents were tested for the preparation of 5-phenyl- $1 \mathrm{H}$ tetrazole(1) from the reaction of benzonitrile with sodium azide in the presence of $2 \mathrm{~mol} \%$ of $\mathrm{CuSO}_{4} \cdot 5 \mathrm{H}_{2} \mathrm{O}$ (Table 1 , entries 5-9). No product was obtained when the reaction was performed in nitromethane, chlorobenzene and anisol (Table 1, entries 5-7). Other solvents, such as water gave the desired product in low yield but NMP gave slightly higher yield (Table 1, entries 8-9). In solvent free condition, 5 -phenyl $1 H$-tetrazole was produced in $50 \%$ yield after long period of time (Table 1, entry 10). As shown in Table 1 among the different solvents tested DMSO was found to be the solvent of choice because of its high dipole moment. The excellent yield was obtained in DMSO at $140{ }^{\circ} \mathrm{C}$ by applying $2 \mathrm{~mol} \%$ of $\mathrm{CuSO}_{4} \cdot 5 \mathrm{H}_{2} \mathrm{O}$ and 1:1 molar ratio of benzonitrile:sodium azide (Table 1, entry 13 ).

To understand the scope and the generality of $\mathrm{CuSO}_{4} \cdot 5 \mathrm{H}_{2} \mathrm{O}$ promoted $(3+2)$ cycloaddition reaction, a variety of structurally divergent benzonitriles possessing a wide range of functional groups was chosen and the results are presented in Table 2 .

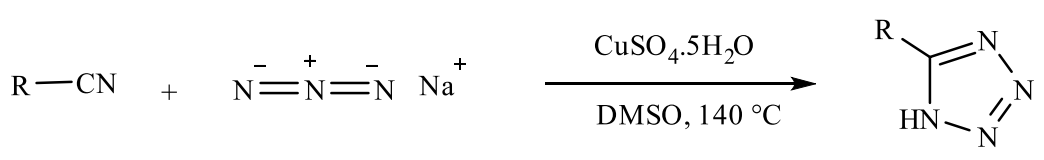

1-17

Scheme 1. Synthesis of 5-phenyl1 $H$-tetrazole (1).

Table 1. Synthesis of 5-phenyl-1H-tetrazole (1) in the presence of different mol $\%$ of $\mathrm{CuSO}_{4} \cdot 5 \mathrm{H}_{2} \mathrm{O}$ in various solvents, different molar ratios of benzonitrile: $\mathrm{NaN}_{3}$ and different temperatures

\begin{tabular}{|c|c|c|c|c|c|c|}
\hline entry & solvent & catalyst & $\begin{array}{c}\text { molar ratio } \\
\text { (benzonitrile: } \mathrm{NaN}_{3} \text { ) }\end{array}$ & time / h & Temp. $/{ }^{\circ} \mathrm{C}$ & conversion / \% \\
\hline 1 & $\mathrm{DMF}$ & none & $1 / 1.5$ & 17 & 120 & 40 \\
\hline 2 & $\mathrm{DMF}$ & $\mathrm{CuSO}_{4} \cdot 5 \mathrm{H}_{2} \mathrm{O}(2 \mathrm{~mol} \%)$ & $1 / 1.5$ & 15 & 120 & 100 \\
\hline 3 & $\mathrm{DMF}$ & $\mathrm{CuSO}_{4} \cdot 5 \mathrm{H}_{2} \mathrm{O}(5 \mathrm{~mol} \%)$ & $1 / 1.5$ & 2 & 120 & 95 \\
\hline 4 & $\mathrm{DMF}$ & $\mathrm{CuSO}_{4} \cdot 5 \mathrm{H}_{2} \mathrm{O}(7 \mathrm{~mol} \%)$ & $1 / 1.5$ & 2 & 120 & 95 \\
\hline 5 & $\mathrm{MeNO}_{2}$ & $\mathrm{CuSO}_{4} \cdot 5 \mathrm{H}_{2} \mathrm{O}(2 \mathrm{~mol} \%)$ & $1 / 1.5$ & 24 & reflux & 0 \\
\hline 6 & $\mathrm{PhCl}$ & $\mathrm{CuSO}_{4} \cdot 5 \mathrm{H}_{2} \mathrm{O}(2 \mathrm{~mol} \%)$ & $1 / 1.5$ & 24 & 120 & 0 \\
\hline 7 & PhOMe & $\mathrm{CuSO}_{4} \cdot 5 \mathrm{H}_{2} \mathrm{O}(2 \mathrm{~mol} \%)$ & $1 / 1.5$ & 24 & 120 & 0 \\
\hline 8 & $\mathrm{H}_{2} \mathrm{O}$ & $\mathrm{CuSO}_{4} \cdot 5 \mathrm{H}_{2} \mathrm{O}(2 \mathrm{~mol} \%)$ & $1 / 1.5$ & 24 & reflux & 45 \\
\hline 9 & NMP & $\mathrm{CuSO}_{4} \cdot 5 \mathrm{H}_{2} \mathrm{O}(2 \mathrm{~mol} \%)$ & $1 / 1.5$ & 6 & 120 & 100 \\
\hline 10 & none & $\mathrm{CuSO}_{4} \cdot 5 \mathrm{H}_{2} \mathrm{O}(2 \mathrm{~mol} \%)$ & $1 / 1.5$ & 20 & 120 & 50 \\
\hline 11 & DMSO & $\mathrm{CuSO}_{4} \cdot 5 \mathrm{H}_{2} \mathrm{O}(2 \mathrm{~mol} \%)$ & $1 / 1.5$ & 3 & 120 & 100 \\
\hline 12 & DMSO & $\mathrm{CuSO}_{4} \cdot 5 \mathrm{H}_{2} \mathrm{O}(2 \mathrm{~mol} \%)$ & $1 / 1.5$ & 1 & 140 & 100 \\
\hline 13 & DMSO & $\mathrm{CuSO}_{4} \cdot 5 \mathrm{H}_{2} \mathrm{O}(2 \mathrm{~mol} \%)$ & $1 / 1$ & 1 & 140 & 100 \\
\hline
\end{tabular}


Table 2. Synthesis of different structurally tetrazoles (1-17) in the presence of $\mathrm{CuSO}_{4} .5 \mathrm{H}_{2} \mathrm{O}$ in DMSO

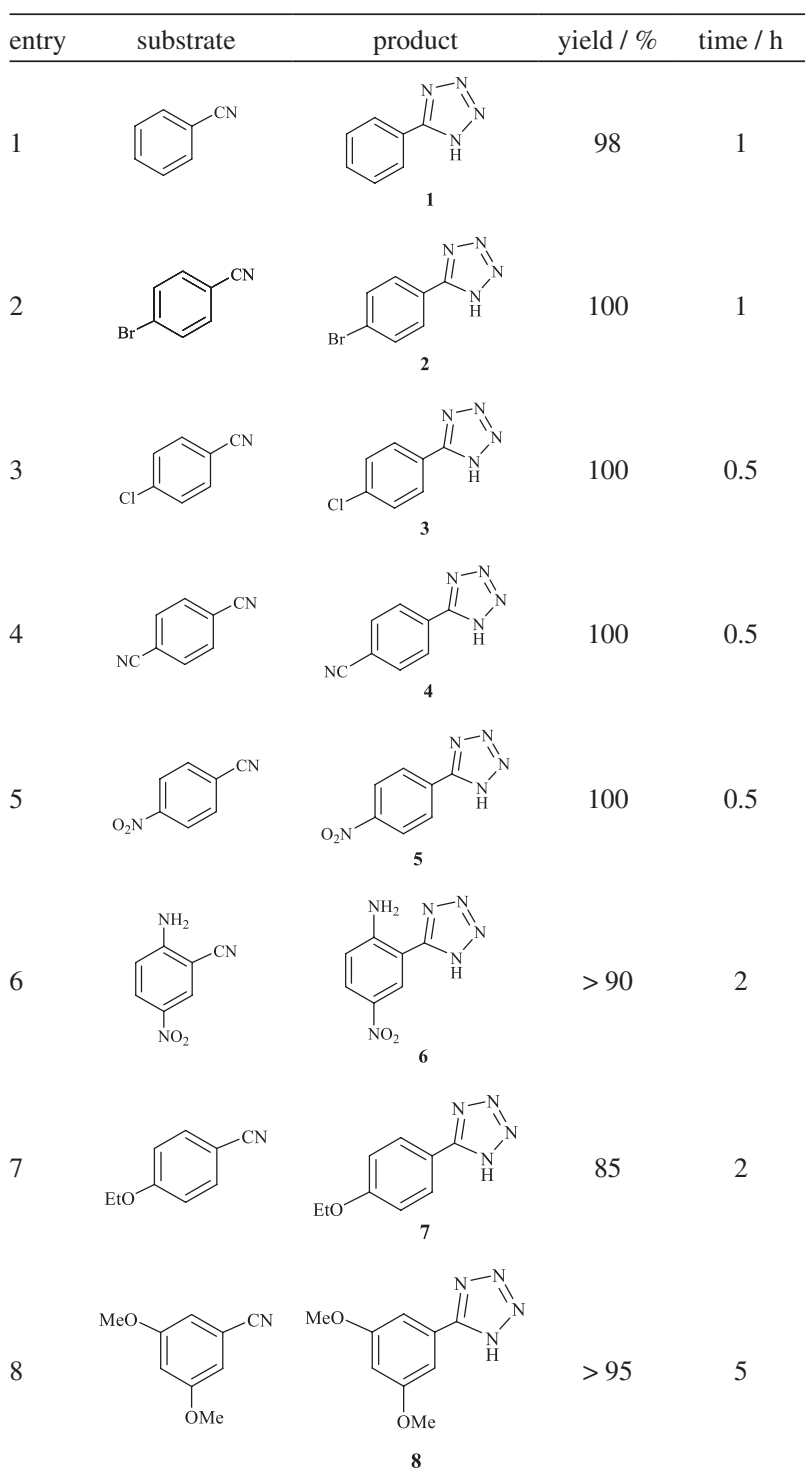

a Reaction temperature: $120^{\circ} \mathrm{C}$.

Among the various nitriles tested, the aromatic nitriles with electron withdrawing substituent gave excellent yields in a very short time (Table 2, entries 2-5). Several nitriles containing electron-donating groups, were successfully converted into their corresponding tetrazoles with a prolonged reaction time (Table 2, entries 6-10). Likewise, aliphatic nitriles react similarly and provide good yields of the corresponding tetrazoles (Table 2, entries 15-17). Heteroaromatic nitriles such as thiophene-2-carbonitrile, 4-pyridinecarbonitrile and 2-pyridinecarbonitrile gave the corresponding tetrazoles in shorter reaction times with excellent yields (Table 2, entries 12-14). It is noteworthy that 1,4-dicyanobenzene only gave mono adduct even by using 1:2 molar ratio of 1,4-dicyanobenzene:sodium azide and $4 \mathrm{~mol} \%$ of catalyst (Table 2, entry 4 ).

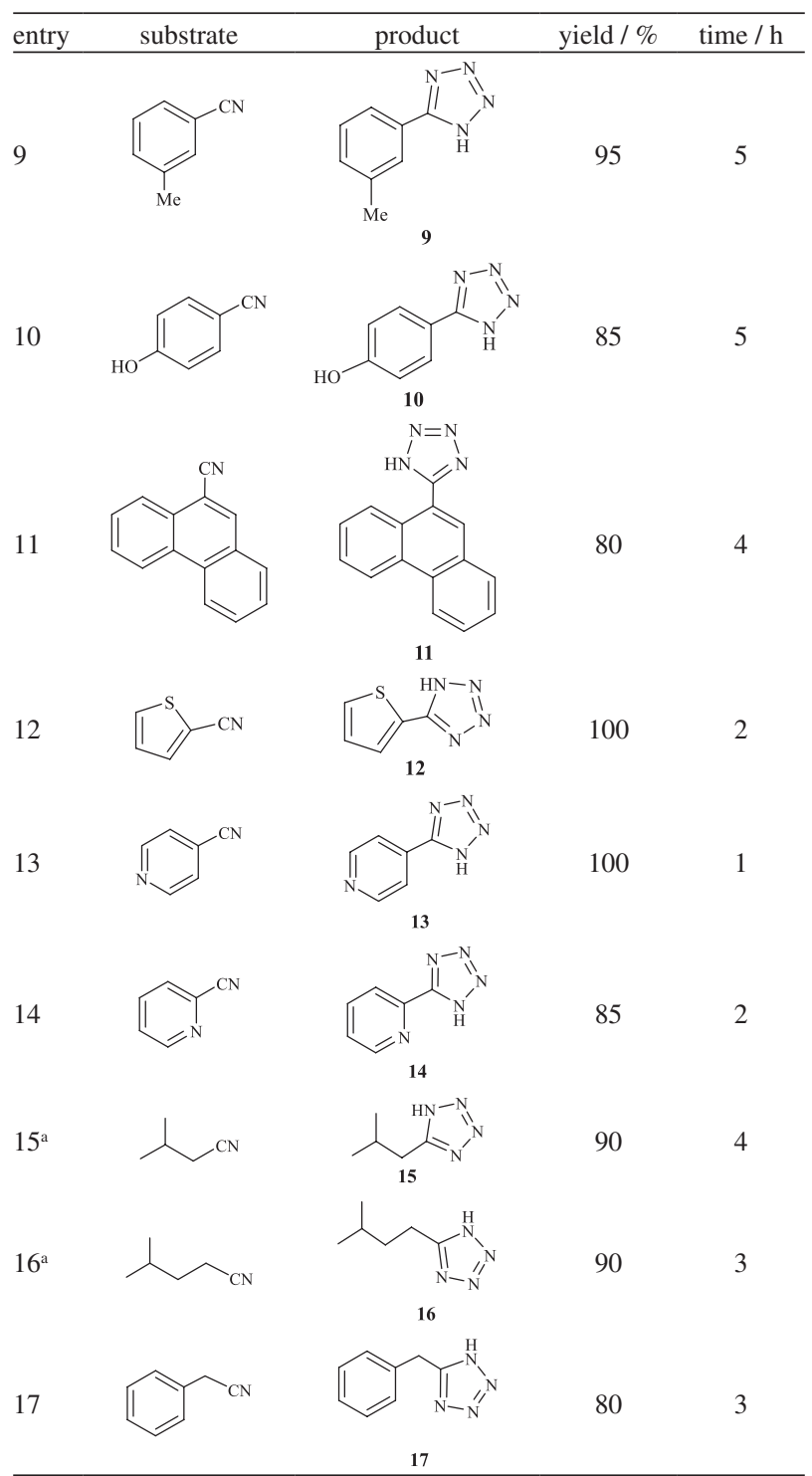

The activity of nitrile compound towards azide ion plays an important role in this cycloaddition reaction. In comparison the cycloaddition reaction of aromatic nitriles with electron withdrawing substituents such as $-\mathrm{Cl}$, $\mathrm{Br},-\mathrm{CN},-\mathrm{NO}_{2}$ and heteroaromatic nitriles compound such as thiophene-2-carbonitrile, 4-pyridinecarbonitrile and 2-pyridinecarbonitrile is faster than the reaction of aromatic nitrile compound with electron donating substituent such as $-\mathrm{NH}_{2},-\mathrm{OCH}_{3},-,-\mathrm{OCH}_{2} \mathrm{CH}_{3}$ and $-\mathrm{OH}$. From Table 2, it is clear that, excellent to good results were obtained with alkyl, aryl and heteroaryl nitriles, despite the different activities of the nitrile derivatives. It seems likely that the high polarity of solvent and efficient catalytic activity of $\mathrm{CuSO}_{4} \cdot 5 \mathrm{H}_{2} \mathrm{O}$ have leveled off the activity of nitrile group. 


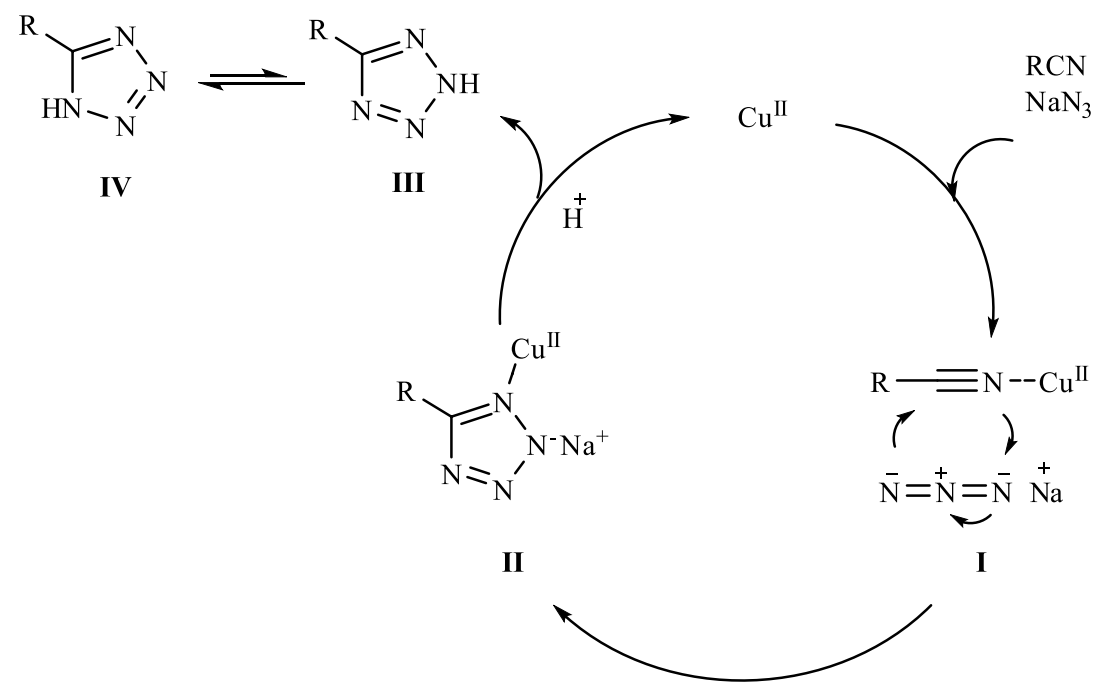

Scheme 2. A plausible mechanism for the formation of tetrazoles.

The structures of all synthesized compounds were confirmed by spectral and analytical data. The IR spectra of all products show absorption bands at 1293-1233 due to $(\mathrm{N}-\mathrm{N}=\mathrm{N}-), 1041-1106$ and $1189-1110$ due to (tetrazole ring). $\mathrm{A}^{13} \mathrm{C}$ NMR signal at $161-154 \mathrm{ppm}$ is assigned to the quaternary carbon of $\mathrm{NH}-\mathrm{C}=\mathrm{N}$.

A plausible mechanism is shown in Scheme 2. Initially, coordination of nitrogen atoms of nitrile compounds with $\mathrm{Cu}^{\mathrm{II}}$ forms complex I which accelerates the cyclization step. This idea is supported by performing the reaction in the absence of $\mathrm{CuSO}_{4} \cdot 5 \mathrm{H}_{2} \mathrm{O}$. Without any catalyst, cycloaddition reaction is not completed even after long period of time (Table1, entry 1 ). The [3+2] cycloaddition between the $\mathrm{C} \equiv \mathrm{N}$ bond of nitrile compound and azide ion takes place readily to form the intermediate II. Acidic work-up, affords III and IV. The equilibrium leads to formation of the more stable tautomer IV (5-substituted $1 H$-tetrazole).

\section{Conclusions}

We have reported an efficient synthetic method for 5 -substituted- $1 H$-tetrazoles by a successive $[3+2]$ cycloaddition of various nitriles with sodium azide in the presence of catalytic amount of $\mathrm{CuSO}_{4} \cdot 5 \mathrm{H}_{2} \mathrm{O}$. This method is applicable to a range of nitriles including aliphatic, aromatic, and heterocyclic nitriles. It has also been shown that, the yields are high and reaction completion time is within $0.5-5 \mathrm{~h}$. The catalyst used is readily available and is environmentally friendly. Short reaction time, good to excellent yields, safe process and simple workup make this method an attractive and useful contribution to the present organic synthesis for the preparation of 5-substituted $1 H$-tetrazoles.

\section{Supplementary Information}

Supplementary information (spectra of synthesized compounds) is available free of charge at http://jbcs.sbq.org.br as PDF file.

\section{Acknowledgments}

The authors gratefully acknowledge the partial support of this study by Ferdowsi University of Mashhad Research Council.

\section{References}

1. Okabayashi, T.; Kano, H.; Makisumi, Y.; Chem. Pharm. Bull. 1960, 8, 157.

2. Sangal, S. K.; Ashok Kumar, A.; J. Indian Chem. Soc. 1986, 63,351 .

3. Witkowski, J. K.; Robins, R. K.; Sidwell, R.W.; Simon, L. N.; J. Med. Chem. 1972, 15, 1150.

4. Tsov, K. C.; Su, H. C. F.; J. Med. Chem. 1963, 6, 693.

5. Bary, V. C.; Conalty, M. C.; O’Sllivan, J. P.; Twomey, D.; Chemotherapy 1977, 8, 103.

6. Maxwell, J. R.; Wasdahl, D. A.; Wolfson, A. C.; Stenberg,V. I.; J. Med. Chem. 1984, 27, 1565.

7. Stewart, K. D.; Bioorg. Med. Chem. Lett. 1998, 529.

8. Shukla, J. S.; Ahmed, J.; Saxena, S.; J. Indian Chem. Soc. 1979, 41,70 .

9. Shishoo, C. J.; Devani, M. B.; Karvekar, M. D.; Vilas,G. V.; Anantham, S.; Bhaati, V. S.; Indian J. Chem., Sect B 1982, 21, 666.

10. Ray, S. M.; Lahiri, S. C.; J. Indian Chem. Soc.1990, 67, 324.

11. Hayao, S.; Havera, H. J.; Strycker, W. G.; Leipzig, T. J.; Rodriguez, R.; J. Med. Chem. 1965, 10, 400. 
12. Fig dor, S. K.; Von Wittenau, M. S.; J. Med. Chem. 1967, 10, 1158.

13. Singh, H.; Chala, A. S.; Kapoor, V. K.; Paul, D.; Malhotra, R. K.; Prog. Med. Chem. 1980, 17, 151.

14. Ostrovskii, V. A.; Pevzner, M. S.; Kofmna, T. P.; Shcherbinin, M. B.; Tselinskii, I.V.; Targets Heterocyclic Syst. 1999, 3, 467.

15. Hiskey, M.; Chavez, D. E.; Naud, D. L.; Son, S. F.; Berghout, H. L.; Bome, C.A.; Proceedings of the International Pyrotechnics Seminars, $27^{\text {th }}$ seminar, Grand Junction, CO, USA, 2000, p. 3.

16. Koldobskii, G. I.; Ostrovskii, V. A.; Usp. Khim. 1994, 63, 847.

17. Huisgen, R.; Sauer, J.; Sturn, H. J.; Markgraf, J. H.; Chem. Ber. 1960, 93, 2106.

18. Moderhack, D.; J. Prakt. Chem. 1988, 340, 687.

19. Hantzsch, A.; Vagt, A.; Justus Liebigs Ann.Chem. 1901, 314, 339.

20. Curran, D. P.; Hadida, S.; Kim, S.-Y.; Tetrahedron 1999, 55, 8997.

21. Kumar, A.; Narayanan, R.; Shechter, H.; J. Org. Chem. 1996, 61,4462 .

22. Koguro, K.; Oga, T.; Mitsui, S.; Orita, R.; Synthesis 1998, 910.

23. Demko, P. Z.; Sharpless, K. B.; J. Org. Chem. 2001, 66, 7945.

24. Himo, F.; Demko, P. Z.; Noodleman, L.; Sharpless, K. B.; J. Am. Chem. Soc 2002, 124, 12210.

25. Bonnamour, J.; Bolm, C.; Chem. Eur. J. 2009, 15, 4543.

26. Amantini, D.; Beleggia, R.; Fringuelli, F.; Pizzo, F.; Vaccoro, L.; J. Org. Chem. 2004, 69, 2896.

27. Yizhong, Z.; Yiming, R.; Chun, C.; Helv. Chim. Acta 2009, 92, 171.

28. Su, W. K.; Hong, Z.; Shan, W. G.; Zhang, X. X.; Eur. J. Org. Chem. 2006, 2723.

29. Hajra, S.; Sinha, D.; Bhowmick, M.; J. Org. Chem. 2007, 72, 1852.

30. Matthews, D. P.; Green, J. E.; Shuker, A. J.; J. Comb. Chem. 2000, $2,19$.

31. Gyoung, Y. S.; Shim, J.-G.; Yamamoto, Y.; Tetrahedron Lett. 2000, 41, 4193.

32. Kantam, M. L.; Shiva Kumar, K. B.; Raja, K. P.; J. Mol. Catal. A: Chem. 2006, 247, 186.

33. Kantam, M. L.; Balasubrahmanyam, V.; Shiva Kumar, K. B.; Synth. Commun. 2006, 36, 1809.

34. Jin, T.; Kitahara, F.; Kamijo, S.; Yamamoto, Y.; Tetrahedron Lett. 2008, 49, 2824.

35. Jin, T.; Kitahara, F.; Kamijo, S.; Yamamoto, Y.; Chem. Asian J. 2008, 3, 1575 .

36. Nasrollahzadeh, M.; Bayat, Y.; Habibi, D.; Moshaee, S.; Tetrahedron Lett. 2009, 50, 4435.
37. Venkateshwarlu, G.; Premalatha, A.; Rajanna, K. C.; Saiprakash, P. K.; Synth. Commun. 2009, 39, 4479.

38. Qi, G.; Dai, Y.; Chin. Chem. Lett. 2010, 21, 1029.

39. Lang, L.; Li, B.; Liu, W.; Jiang, L.; Xu, Z.; Yin, G.; Chem. Commun. 2010, 46, 448.

40. Nasrollahzadeh, M.; Habibi, D.; Shahkarami, Z.; Bayat, Y.; Tetrahedron 2009, 65, 10715.

41 Ben Achma, R.; Ghorbel, A.; Dafinov, A.; Medina, F.; Appl. Catal., A 2009, 349, 20.

42. Varma, R. S.; Tetrahedron 2002, 58, 1235.

43. Shinde, A. B.; Shrigadi, N. B.; Samant, S. D.; Appl. Catal., A 2004, 276, 5.

44. Clark, P. D.; Mesher, S. T. E.; Primak, A.; Yao, H.; Catal. Lett. 1997, 43, 79.

45. Yin, W.; Shi, M.; Tetrahedron 2005, 61, 10861.

46. Gue'lou, E.; Barrault, J.; Fournier, J.; Tatibouet, J. M.; Appl. Catal., B 2003, 44, 1.

47. Caudo, S.; Centi, G.; Genovese, C.; Perathoner, S.; Appl. Catal., B 2007, 70, 437.

48. Rama,V.; Kanagaraj, K.; Pitchumani, K.; J. Org. Chem. 2011, 76,9090

49. Sheldon, R. A.; J. Chem. Technol. Biotechnol. 1997, 68, 381.

50. Nandre, K. P.; Salunke, J. K.; Nandre, J. P.; Patil,A. U.; Borse,V. S.; Bhosale, S. V.; Chin. Chem. Lett. 2012, 23, 161.

51. Akhlaghinia, B.; Tavakoli, S.; Synthesis 2005, 1775.

52. Aureggi, V.; Sedelmeier, G.; Angew. Chem., Int. Ed. 2007, 46, 8440.

53. Lossen,S.; Ann. Chem. 1897, 298, 102.

54. Köennecke, A.; Döme, R.; Kleinpeter, E.; Lippmann, E.; Tetrahedron 1997, 35, 1957.

55. Tienan, J.; Fukuzou, K.; Shin, K.; Yoshinori, Y.; Tetrahedron Lett. 2008, 49, 2824.

56. Ek, F.; Wistrand, L.-G.; Frejd, T.; J. Org. Chem. 2003, 68, 1911.

57. Sreedhar, B.; Kumar, A. S.; Yada, D.; Tetrahedron Lett. 2011, 52,3565 .

58. Satzinger, G.; Justus Liebigs Ann. Chem. 1960, 638, 159.

59. Najafi Chermahini, A.; Teimouri, A.; Moaddeli, A.; Heteroat. Chem. 2011, 22, 168.

60. Herbst, M.; J. Org. Chem. 1950, 15, 1082.

61. Katritzky, A. R.; El-Gendy, B. E. M.; Draghici, B.; Hall, C. D.; Steel, P. J.; J. Org. Chem. 2010, 75, 6468.

Submitted: August 30, 2012

Published online: January 9, 2013 